\title{
Mirada a los clásicos. "Cine fórum”, nuevo enfoque interdisciplinar en el Grado de Óptica y Optometría A look back at the classics."Cinema forum", new interdisciplinary approach in the Optics and Optometry Degree
}

\author{
María José Luesma ${ }^{1}$, Fernando Soteras ${ }^{2}$, Ana Rosa Abadía ${ }^{2}$ \\ mjluesma@unizar.es, fsoteras@unizar.es, arabad@unizar.es \\ ${ }^{1}$ Departamento Anatomía e Histología Humanas \\ Universidad de Zaragoza \\ Zaragoza, España \\ ${ }^{2}$ Departamento Farmacología y Fisiología \\ Universidad de Zaragoza \\ Zaragoza, España
}

Resumen- Hace ya cinco cursos académicos se inició una colaboración interdisciplinar entre dos asignaturas pertenecientes a áreas de conocimiento distintas y que se imparten en el primer curso del Grado en Óptica y Optometría de la Universidad de Zaragoza; dicha colaboración, se centra en el Aprendizaje Basado en Problemas (ABP) y en la elaboración de Portafolios. Con el propósito de explorar nuevas estrategias educativas que permitan abordar con carácter integrador ambas asignaturas, y sin abandonar las que ya tenemos integradas en la docencia de nuestras asignaturas, hemos utilizado, durante el presente curso académico 2016-17, el cine como recurso formativo; obteniendo unos resultados claramente satisfactorios puesto que los alumnos además de trabajar aspectos puramente técnicos de las materias, apreciaron aprendizaje en lo que se refiere a aspectos más emocionales, afectivos o empáticos como futuros profesionales sanitarios.

Palabras clave: Interdisciplinariedad, Cine formativo, Cine y Salud.

Abstract- Five academic years ago, an interdisciplinary collaboration started between two subjects belonging to different areas of knowledge in the first year of the Optics and Optometry Degree at the University of Zaragoza. This collaboration focuses on Problem-Based Learning ( $\mathrm{PbL}$ ) and Portfolios methodologies. With the purpose of exploring new educational strategies that allow us to approach both subjects comprehensively, and without abandoning those that we already have integrated in the teaching of our subjects, we have used, during the present academic year 2016-17, the cinema as a formative resource; obtaining clearly satisfactory results since the students, besides working purely technical aspects of the subjects, appreciated learning in terms of more emotional, affective or empathic aspects as future health professionals.

Keywords: Interdisciplinarity, Educational cinema, Film and Health.

\section{INTRODUCCIÓN}

Las innovaciones educativas, además de ser eficaces, eficientes y transferibles, deben de ser consolidables incluso cuando la fuente de financiación para su implementación haya cesado (Fidalgo 2017). Este ha sido uno de nuestros objetivos prioritarios desde la creación de la colaboración interdisciplinar, hace ya 5 cursos académicos, entre las asignaturas Anatomía e Histología Ocular y Fisiología Ocular y del Sistema Visual impartidas en el primer curso del grado de Óptica y Optometría; colaboración que se estableció desde su origen con vocación de permanencia.

Nuestra concepción de innovación no es de un elemento estático sino que tal y como defiende Jaume Carbonell (2002) "la innovación no es una actividad puntual sino un proceso, un largo viaje o trayecto que se detiene a contemplar la vida en las aulas, la organización de los centros, la dinámica de la comunidad educativa y la cultura profesional del profesorado. $\mathrm{Su}$ propósito es alterar la realidad vigente, modificando concepciones y actitudes, alterando métodos e intervenciones y mejorando o transformando, según los casos, los procesos de enseñanza y aprendizaje. La innovación, por tanto, va asociada al cambio". Este ha sido el leitmotiv desde nuestros orígenes, lo que ha supuesto la concatenación de múltiples proyectos docentes que no han sido más que la evolución de uno primigenio sometido a constante evaluación, revisión y reajuste. Siempre atentos a los resultados obtenidos con cada cambio, hemos adoptado medidas correctoras para subsanar los defectos encontrados o incorporado nuevas propuestas y estrategias que nos han permitido abordar nuevos objetivos y retos en el proceso de enseñanza-aprendizaje de nuestras asignaturas, de una manera conjunta.

Dentro de esta búsqueda de nuevas metodologías, el cine fórum, aún sin ser una estrategia novedosa, nos permite trabajar de una manera interdisciplinar competencias específicas y transversales de dos asignaturas de carácter biomédico, lo que aporta una versión actualizada de la misma (Baños et al. 2005).

En tanto en cuanto el Graduado en Óptica y Optometría es considerado como profesional sanitario, buscamos un complemento humano al conocimiento más técnico estudiado durante el curso académico, de esta manera, y conforme a lo propuesto por Loscos et al. (2006), el cine justificaría su función docente desde cuatro perspectivas diferentes: la función diseminadora de las situaciones relacionadas con la enfermedad; el desarrollo de un razonamiento crítico, la estimulación del conocimiento emocional de la enfermedad, y el conocimiento de las consecuencias sociales e individuales de la enfermedad. 
Por otra parte, y teniendo en cuenta que el lenguaje cinematográfico cuenta con niveles distintos de comunicación tal como sintetiza Icart-Isern et al. (2008): Un primer nivel perceptivo (lo que se ve y se oye); un segundo nivel que corresponde a los lenguajes asociados (los referentes culturales), y un último y más complejo nivel subconsciente (las sensaciones y las emociones); el cine va a facilitar a nuestros alumnos un mayor aprendizaje significativo del impacto emocional y social de la enfermedad asociada al sistema visual al que se someterán en su futura profesión.

El visionado de películas comerciales y su posterior debate dirigido se trata de una experiencia lúdica que, con la actual terminología, podemos enmarcar en el campo de la gamificación o ludificación. Según Marín y Hierro (2013) "la gamificación es una técnica, un método y una estrategia a la vez. Parte del conocimiento de los elementos que hacen atractivos a los juegos e identifica, dentro de una actividad, tarea o mensaje determinado, en un entorno de NO-juego, aquellos aspectos susceptibles de ser convertidos en juego o dinámicas lúdicas. Todo ello para conseguir una vinculación especial con los usuarios, incentivar un cambio de comportamiento o transmitir un mensaje o contenido. Es decir, crear una experiencia significativa y motivadora" (LlorensLargo et al. 2015).

No existe la menor duda de que los estudiantes universitarios se sienten atraídos por el cine, y que las películas comerciales son una herramienta muy eficaz para ofrecerles escenarios convincentes sobre situaciones consideradas de interés por los profesores (Baños et al. 2005).

\section{CONTEXTO}

Los estudiantes que cursan simultáneamente las asignaturas de Anatomía e Histología Ocular y Fisiología Ocular y del Sistema Visual, realizan actividades comunes utilizando las metodologías del Aprendizaje Basado en Problemas (ABP) y la elaboración de Portafolios. Ambas asignaturas se imparten en el primer curso académico del Grado en Óptica y Optometría en la Facultad de Ciencias de la Universidad de Zaragoza. Durante los primeros años, la colaboración se centró en la adquisición de competencias específicas y genéricas de ambas asignaturas desde una perspectiva interdisciplinar. Pozuelos et al. (2012) aluden al "potencial que encierra desarrollar experiencias en las que los contenidos se conecten y presenten de manera que hagan referencia a situaciones reales y plenas de sentido ganándose, de esta forma, en comprensión y funcionalidad” (Pozuelos et al. 2012). Esta interdisciplinariedad a través del ABP, ha facilitado la interacción entre ambas asignaturas para un conocimiento más integral. En lo que se refiere a las competencias genéricas se hecho especial hincapié en todas aquellas que se desarrollan dentro del trabajo cooperativo (reparto de roles y responsabilidades, aprendizaje autónomo, gestión del tiempo, resolución de conflictos, tolerancia a la frustración, etc.). Esta experiencia continuada ha sido objeto de múltiples Proyectos de Innovación Docente que han sido plasmados en diversas publicaciones (Luesma et al. 2014a, 2014b, 2015a, 2015b, 2015c, 2015d, 2016). En este nuevo reto, como continuidad a todos los anteriores y, dado que La ley 44/2003 de Ordenación de las Profesiones Sanitarias reconoce al Graduado en Óptica y Optometría como un profesional sanitario, se ha propuesto trabajar aspectos básicos sociales de su futura carrera profesional dentro del marco de la sanidad, sin abandonar todas aquellas competencias mencionadas anteriormente. Con el fin de propiciar un debate sobre las implicaciones sociales de la enfermedad asociada a la función visual y también las propias de su futuro trabajo, se ha planteado recuperar una vieja estrategia como es la utilización del cine, para analizar causas y consecuencias de la enfermedad a través de la visualización de fragmentos de películas. A esta modalidad de cine fórum se ha pretendido darle un nuevo enfoque más actual de interdisciplinariedad a través del trabajo de competencias transversales tales como el pensamiento crítico, el aspecto humano de la enfermedad, las implicaciones sociales y personales, etc.

\section{A. Objetivos de la experiencia}

- Explorar otras estrategias que, desarrolladas conjuntamente, nos permitan integrar la docencia de ambas asignaturas; filosofía que inspira nuestro proyecto desde sus inicios.

- Impartir de manera integrada e interdisciplinarmente contenidos propios de las asignaturas utilizando fragmentos de películas y un debate posterior moderado por el profesorado de ambas asignaturas (trabajando competencias conceptuales propias de las asignaturas)

- $\quad$ Fomentar la reflexión y el pensamiento crítico de la enfermedad desde un punto de vista social a través de películas (trabajando competencias genéricas)

- Analizar la utilización de esta estrategia desde la perspectiva de adquisición de competencias.

\section{DESCRIPCIÓN}

Al inicio del curso académico, los responsables de ambas asignaturas realizaron una selección de películas y fragmentos de películas comerciales que guardan relación con los objetivos previstos en el apartado anterior. Finalmente se decidió la proyección de fragmentos de la película danesa titulada "Bailar en la Oscuridad" dirigida por Lars Von Trier en el año 2000, protagonizada por Björk y Catherine Deneuve entre otros. Se trata de un drama musical enmarcada dentro de la corriente "dogma 95". Tal como sugieren Baños et al. (2005) no se trata de una pieza muy conocida por el público joven, para no restar interés por la misma. El argumento gira en torno a la lucha de una inmigrante checa en los Estados Unidos, contra una enfermedad degenerativa hereditaria que ella padece y que ha transmitido a su hijo; enfermedad que irremediablemente conduce a la ceguera.

La actividad se organizó en una sesión dentro de los seminarios propuestos en el año académico. La duración de las piezas a visionar no fue muy extensa para no restar tiempo al debate; de las dos horas y media que duró la actividad, en torno al $50 \%$ se dedicó a la visualización de los fragmentos y el 50\% restante a la presentación inicial y debate posterior de los mismos. Antes de la proyección se informó a los estudiantes en qué consistiría la actividad: la película seleccionada, su duración, el debate posterior al visionado, la necesidad de elaborar un pequeño informe tras haber reflexionado y discutido en pequeños grupos todo lo que les hubiera supuesto, etc. Además se intentó consensuar lo máximo posible la fecha en que se iba a realizar la proyección, para dar mayor relevancia e importancia a la actividad facilitando la asistencia del mayor número posible de alumnos. El día de la proyección, tras presentar brevemente las 
características técnicas y contextuales de la película, 60 estudiantes matriculados simultáneamente en las asignaturas de Anatomía e Histología Ocular y Fisiología Ocular de primer curso del Grado en Óptica y Optometría visualizaron fragmentos de la misma en un aula dotada de reproductor de DVD y un sistema de proyección. Tras ver las piezas seleccionadas, se procedió a un debate moderado y guiado a través de preguntas propuestas por el profesorado de ambas asignaturas, estimulándose de igual manera la participación libre de los estudiantes. Durante la discusión se intentó encontrar un diagnóstico posible a la enfermedad padecida por nuestra protagonista, ya que en ninguna parte se dice de manera explícita cual es exactamente, de tal manera que pudimos abordar la enfermedad desde un punto de vista anatomo-fisiológico recordando y plantando cuestiones más técnicas dentro de nuestros ámbitos académicos; si bien es verdad que incidimos especialmente en aquellos aspectos que facilitan el aprendizaje relacionado con la afectividad, la emotividad y la empatía ante la enfermedad, desde un punto de vista del profesional sanitario. Al finalizar la actividad y antes de abandonar la sala, se produjo otro pequeño debate por un grupo considerablemente numeroso de 12 alumnos, más informal e intimista. Hay muchas personas que por timidez no son proclives a expresar sus opiniones y sentimientos en grandes grupos, es por ello que, tras el debate los alumnos realizaron un pequeño informe (competencia genérica importante) con aquellos aspectos que más les hubieran interesado, sorprendido o estimulado de alguna manera; dicho informe fue realizado por grupos, aquellos coincidentes con la metodología del ABP. El informe se realizó en otro momento diferente al día de celebración del cine fórum, con el fin de dejar reposar la película, los elementos que salieron en el debate y propiciar primero una reflexión más personal y luego otro más grupal. Para su elaboración se les facilitó una brevísima guía que incluía las siguientes preguntas:

1. ¿Cómo valoraríais la película? ¿Qué os ha parecido con carácter general?

\section{2. ¿Qué temas o conflictos se planean en la película?}

3. ¿ ¿Habéis descubierto algún aspecto de la Enfermedad (además de la propia enfermedad en sí misma) que no os hubieseis planteado nunca? ¿Cuáles?

Al concluir la experiencia completa y tras haber realizado los informes por grupos, los alumnos respondieron, de manera individual, a una encuesta de satisfacción que permitiera testar la nueva estrategia interdisciplinar realizada; constaba de cuatro preguntas, en las tres primeras las respuestas variaban del 1-5 (1 = MUY POCO; 2 = POCO; 3 = NORMAL; $4=$ BASTANTE; 5 = MUCHO) mientras que la cuarta pregunta era de respuesta abierta:

\section{1. $\quad \dot{¿}$ Te ha parecido interesante la actividad realizada?}

2. ¿ ¿Has descubierto aspectos de la profesión o de la enfermedad visual que no te hubieras plantado? (en este caso la pregunta es cerrada: análisis cuantitativo)

3. ¿ ¿Te ha parecido útil como sistema de aprendizaje?

4. ¿Qué aspectos positivos y negativos destacarías?

\section{RESUltados}

La actividad fue acogida mayoritariamente con entusiasmo pues la generalidad de los jóvenes disfruta con el cine.
Inicialmente lo consideraron como un entretenimiento y una evasión dentro de otras metodologías que pueden resultar más exigentes. El dramatismo de la película y la narración de un mundo irreal como si fuera real, acercó de manera contundente las consecuencias que conlleva la "Enfermedad" con mayúsculas. Fue sorprendente la conmoción que produjo el desenlace de la película que dejó un silencio mantenido en la sala durante unos minutos. Quizás fue el primer resultado observado, difícilmente cuantificable pero locuaz por sí mismo; algo se había movido en su interior; hay películas que son didácticas por sí mismas, y ésta es una de ellas. El debate surgió unos minutos más tarde de manera fluida, en él se analizaron los diferentes elementos de la película.

Tras la actividad en sí misma, recogimos todos los elementos que fueron apareciendo, tanto en el debate como en los informes presentados por los alumnos, y analizamos la información obtenida. Con respecto a la valoración general de la película, cabe destacar que muchos de los alumnos afirmaron que el melodrama no es un género que suelan escoger cuando van al cine, por lo que no están acostumbrados al mismo. De ahí se deduce la importancia de generar oportunidades que les permitan acercarse a este tipo de cine desde otra perspectiva más reflexiva.

Con respecto a los temas y conflictos que plantea la película, fueron múltiples los que se recogieron, dando cabida a la discusión y reflexión de elementos tan importantes como la discapacidad, la aceptación de la misma, su superación, la reorganización sensorial, la deshumanización de la sociedad, la dignificación de la enfermedad, el reconocimiento de la salud como derecho fundamental y universal, la discriminación asociada a la discapacidad y a la inmigración, la maternidad y la paternidad dentro de la discapacidad, el modelo sanitario público o privado, la amistad, la empatía, la solidaridad, la resiliencia, y un largo etc. que nos permite asegurar el haber cumplido los objetivos planteados al inicio del proyecto. El enfoque de interdisciplinariedad entre las asignaturas que forman este proyecto ya consolidado, permite una visión global de todos estos aspectos que alcanzan una dimensión mayor, a la altura de las competencias abordadas en la actividad.

Un aspecto, aunque anecdótico, significativo, es el hecho de que múltiples de los informes solicitados a los grupos fueron presentados manuscritos en una era en la que nuestros estudiantes tienen una gran dependencia de las tecnologías. De manera intuitiva, al plasmar sus pensamientos y reflexiones más personales, han utilizado un sistema de narración mucho más intimista, como es una redacción manuscrita, claro indicio de un trabajo en un plano más afectivo y emocional.

Al finalizar la actividad, y después de presentar sus informes por grupos, 53 alumnos (el 88,3\% de los alumnos que participaron en la actividad) contestaron a la encuesta de satisfacción individual descrita en el apartado 3 del presente documento. De la misma podemos recoger, de una manera objetiva, la siguiente información en relación a las tres primeras preguntas de naturaleza cerrada: el $84.9 \%$ de los alumnos encontraron bastante o muy interesante la actividad realizada, un 69.8 \% afirmaron haber descubierto bastantes o muchos aspectos de la enfermedad sobre los que no habían reflexionado con anterioridad y un 69.8 \% consideraron el cine fórum como un sistema de aprendizaje útil. Sólo un porcentaje entre el 3.8 y el $17 \%$ consideraron la actividad muy poco o poco interesante, útil como sistema de aprendizaje o como 
medio para descubrir aspectos desconocidos de la enfermedad/profesión. Datos reflejados de manera gráfica en la Figura 1.
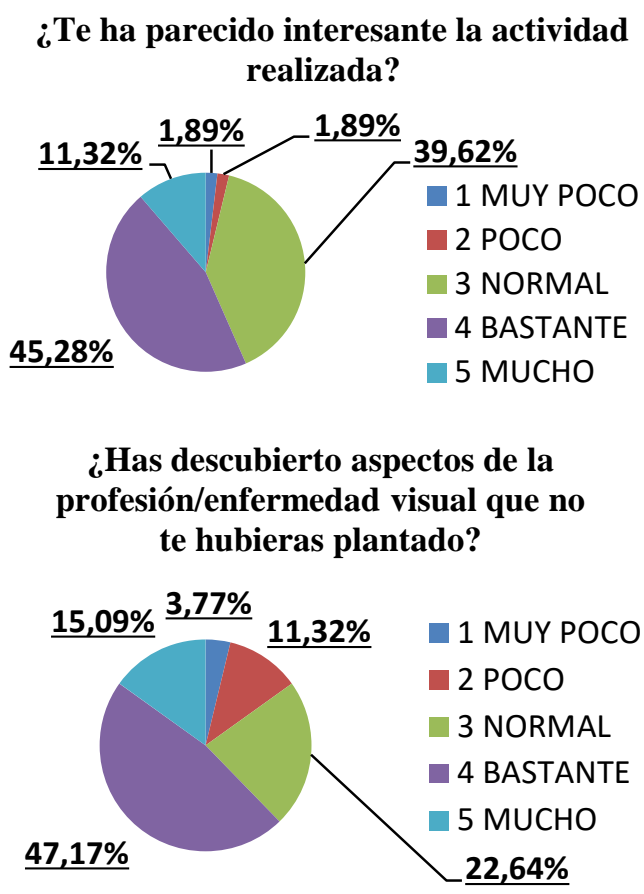

\section{¿Te ha parecido útil como sistema de aprendizaje?}

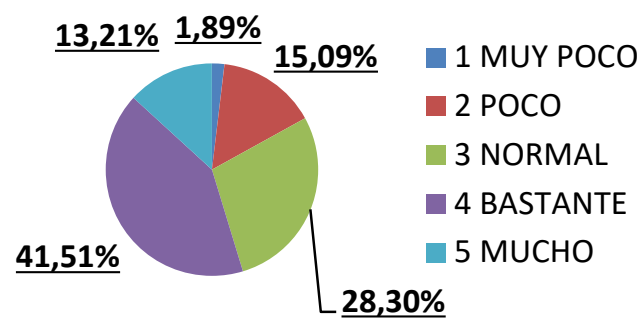

Figura 1. Resultados de la encuesta de satisfacción

Con relación a la cuarta pregunta abierta "¿Qué aspectos positivos y negativos destacarías?”, destacar por encima de todo, la valoración de la actividad en su conjunto por parte de los estudiantes como una estrategia educativa "diferente, motivadora, amena, divertida que fomenta la participación" [Sic] a pesar de la dureza de la película. Se valora muy positivamente el trabajo en grupo mantenido en el debate posterior a la visualización de los fragmentos, también el realizado en equipos para la elaboración de los informes, ya que aunque habiendo trabajado por grupos durante el curso académico en el desarrollo de la metodología del ABP, en esta ocasión lo hacían desde un plano más emocional; valorado muy positivamente la empatía surgida entre ellos e incluso con el profesorado pues "se desarrolla el aspecto más personal" [Sic]. Se propone incluso repetir la actividad en más ocasiones, al menos una vez por semestre.

Tras analizar los aspectos positivos vemos como destacan, de manera recurrente, muchos de los que ya salieron en el debate y en los informes por grupos, tales como el descubrir y reflexionar aspectos que no se habían planteado en torno a la enfermedad, relatan cómo han sentido un cambio en el orden de sus prioridades, como han desarrollado una incipiente empatía por los que serán sus futuros pacientes, etc.

En relación a los aspectos negativos recogidos en la encuesta, destacar sobre todo aspectos logísticos de adecuación de horarios, fechas y ubicación de la actividad. Por motivos propios de la organización académica ha sido muy difícil encontrar un día y hora en la que la mayoría pudieran acudir, ya que es un grado en el que la carga de actividades prácticas es elevado y por lo tanto todos los días de la semana unos grupos u otros tienen prácticas de alguna disciplina; a esto hay que sumar que la actividad debía realizarse en un aula dotada de una determinada infraestructura de audio y proyección, bastante solicitada. Todos estos factores han hecho que la actividad se haya retrasado en el calendario académico casi al final del curso, momento en el que los factores tiempo y disponibilidad adquieren una especial relevancia. Aunque hay que señalar que han valorado positivamente la flexibilidad planteada por el profesorado cambiando la actividad en múltiples ocasiones con el fin de adecuarla a sus necesidades y demandas. Por otra parte y bajo esta perspectiva, algunos estudiantes han considerado la actividad excesivamente larga (duración de 2 horas 30 minutos).

En relación a la película se ha considerado como aspecto negativo su género ya que el melodrama no es de la preferencia de un público joven, y la complejidad que en determinados momentos muestra. También consideran como negativos la "crudeza de la película y el descubrimiento de la crueldad de la enfermedad" [Sic]. Sin pretender desacreditar su opinión, este extremo no lo podemos juzgar como estrictamente negativo ya que uno de nuestros objetivos era precisamente acercarles a esa otra realidad sobre la enfermedad, más allá de la puramente técnica. En este mismo contexto, y por lo tanto no sorprende, reivindican el haber tratado con mayor profundidad el aspecto diagnóstico y clínico de la enfermedad. Si bien es verdad que aunque se aborda el problema clínico, es lo suficientemente abierto como para que pase a un segundo plano, dándole protagonismo a la repercusión que la enfermedad tiene en la vida particular, social y profesional del paciente o del discapacitado; siendo éste el enfoque perseguido desde el inicio de la actividad.

Para finalizar este bloque, tanto en las preguntas abiertas que guiaron el informe, como en las preguntas cerradas realizadas de manera individual, es paradójico observar como algunos de los alumnos que han valorado el cine fórum como sistema de aprendizaje poco útil, a la vez confirman haber descubierto bastantes o muchos aspectos de su profesión sanitaria. Son alumnos de primer curso y quizás no hayamos sido capaces de transmitirles el concepto de formación integral en el que las competencias transversales adquieren la misma importancia que las específicas de nuestras materias.

\section{CONCLUSIONES}

Nuestro proyecto de colaboración interdisciplinar entre ambas asignaturas, que se desarrolla ya durante 5 años académicos, ha ido evolucionando y redefiniéndose constantemente producto de una evaluación exhaustiva de los resultados obtenidos a tenor de los objetivos propuestos en el inicio de cada curso. En una exploración constante de nuevas estrategias educativas que permitan ahondar en nuestro objetivo prioritario del cual emanan todos los demás, la 
continuidad en el enfoque interdisciplinar de nuestras asignaturas, consideramos que el recuperar un clásico: el cine fórum como una herramienta más de integración de conocimiento, ha permitido de manera magistral el abordar no solo aspectos específicos de nuestras disciplinas, sino el trabajar aspectos más personales dentro de un marco de pensamiento crítico que nos permite establecer un aprendizaje significativo emocional en lo concerniente a la enfermedad en tanto profesionales sanitarios.

Por otra parte, la experiencia que se propone en este proyecto es transferible a muchas otras áreas de conocimiento; en tanto que medio de comunicación, el cine puede mostrarnos realidades abarcables por multitud de disciplinas.

En cuanto a la sostenibilidad, todas las metodologías y estrategias utilizadas por este grupo: ABP, elaboración de Portafolios, utilización de rúbricas, utilización de la herramienta docente interactiva SOCRATIVE de Tutoría grupal y, en este caso, visionado de fragmentos de películas, no suponen la necesidad de inversión económica, ni de recursos materiales especiales, ya que actualmente la inmensa mayoría de las aulas disponen de conexión a red, reproductores de DVD, equipos de sonido y proyectores, por lo que consideramos que es sostenible.

"La fotografía es verdad. Y el cine es una verdad 24 veces por segundo” Jean-Luc Godard.

\section{AgRADECIMIENTOS}

Ana Rosa Abadía es coordinadora del Grupo de Investigación en Docencia e Innovación Universitaria (GIDIU) reconocido por el Gobierno de Aragón y financiado por el Fondo Social Europeo.A la Universidad de Zaragoza por la concesión del Proyecto de Innovación Docente PIIDUZ_16_072. A los estudiantes matriculados en las asignaturas de “Anatomía e Histología Ocular” y "Fisiología Ocular y del Sistema Visual” del Grado de Óptica y Optometría de la Universidad de Zaragoza.

\section{REFERENCIAS}

Baños J.E., Aramburu J.F., \& Sentí M. (2005). "Biocinema: la experiencia de emplear películas comerciales con estudiantes de Biología”. Rev Med Cine 1: 42-46.

Carbonell J. (2002). La Innovación Educativa. Cañal de León: 11-12. Madrid: Akal.

Fidalgo A. (2017). Consolidación de la innovación educativa ¿Qué es y cómo se puede conseguir? Recuperado de https://innovacioneducativa.wordpress.com/2017/03/13/c onsolidacion-de-la-innovacion-educativa-que-es-ycomo-se-puede-conseguir/

Icart-Isern M.T., Pulpón A.M., Álvarez R., Barrachina L., Bernat R., Colina J., Isla P. \& SanFeliu V. (2008). Metodología de la investigación y cine comercial: claves de una experiencia docente. Educ Med. 11(1): 13-18.

Llorens-Largo F., Gallego-Durán F.J., Villagrá-Arnedo C.J., Compañ-Rosique P., Satorre-Cuerda R. \& MolinaCarmona R. (2015). Lecciones aprendidas gamificando cuando aún no se llamaba gamificación. Libro de Abstracts del III Congreso Internacional sobre Aprendizaje, Innovación y Competitividad (CINAIC 2015).
Loscos J., Baños J.E., Loscos F. \& de la Cámara J. (2006). Medicine, cinema and literature: A teaching experiment at the Universitat Autónoma de Barcelona. J Med Mov 2: 138-142.

Luesma, M.J., Soteras, F. \& Abadía, A.R. (2014a). Una experiencia de docencia integrada. TESI, 15(3), 36-55.

Luesma M.J. \& Soteras F. (2014b). Proyecto docente interdisciplinar entre las materias de Anatomía, Histología y Fisiología Ocular en el Grado de Óptica Optometría fundamentado en el aprendizaje basado en problemas y en el aprendizaje cooperativo en Experiencias e Innovación docente en el contexto actual de la docencia Universitaria. Ed, Educación Editora. pp: 291-295. ISBN: 978-84-15524-14-4.

Luesma M.J., Soteras F. \& Abadía A.R. (2015a). Colaboración interdisciplinar en el Grado de ÓpticaOptometría en el trabajo en equipo, una herramienta para el aprendizaje. Ed, Prensas Universitarias UZ. pp: 186191. ISBN: 978-84-606-6049-1.

Luesma M.J., Soteras F. \& Abadía A.R. (2015b). Inicio de un aprendizaje integrado: Una Rúbrica de evaluación común. Starting an integrated learning: a common assessment Rubric en XI Foro Internacional sobre Evaluación de la Calidad de la Investigación y la Educación Superior. pp: 400-406. ISBN - 14: 978-84608-7205-4.

Luesma M.J., Soteras F. \& Abadía A.R. (2015c). Evaluación mediante rúbricas de trabajos interdisciplinarios en Los retos de mejorar la evaluación: V Congreso Internacional UNIVEST'15.Servicio de Publicaciones de la Universidad de Girona, pp: 155-159. ISBN: 978-84-845845-37.

Luesma M.J., Soteras F. \& Abadía A.R. (2015d). Análisis de la información de las Actas de las reuniones de trabajo en equipo de los estudiantes en $\mathrm{La}$ sociedad del Aprendizaje. Actas del III Congreso Internacional sobre Aprendizaje, Innovación y Competitividad. CINAIC 2015. Ed, Fundación General de la Universidad Politécnica de Madrid, pp: 429-433. ISBN: 978-84-6082907-2.

Luesma M.J., Soteras F., \& Abadía A.R. (2016). Valoración de la utilización de SOCRATIVE como herramienta didáctica interactiva en dos asignaturas del grado en Óptica-Optometría de la Universidad de Zaragoza” Congreso Nacional de Innovación Educativa y de Docencia en Red. IN_RED 2016. Valencia. Publicado en las Actas del Congreso. Editorial Universitat Politècnica de València. ISBN: 978-84-9048-541-5 (versión cd) DOI: http://dx.doi.org/10.4995/INRED2016.2016.4430

Marín I. \& Hierro E. (2013). Gamificación. El poder del juego en la gestión empresarial y la conexión con los clientes. Empresa Activa.

Pozuelos F.J., Rodríguez, F.P. \& Travé, G. (2012). El enfoque interdisciplinar en la enseñanza universitaria y aprendizaje basado en la investigación. Un estudio de caso en el marco de la formación. Revista de Educación, 357, 561-585. 